\title{
A new functional method to choose the target lobe for lung volume reduction in emphysema - comparison with the conventional densitometric method
}

\author{
This article was published in the following Dove Press journal: \\ International Journal of COPD \\ 30 August 2017 \\ Number of times this article has been viewed
}

Juergen Hetzel,' Michael

Boeckeler,' Marius Horger, ${ }^{2}$

Ahmed Ehab,' Christopher

Kloth, ${ }^{2}$ Robert Wagner, ${ }^{3}$

Lutz Freitag, ${ }^{4}$ Dirk-Jan

Slebos, ${ }^{5}$ Richard Alexander

Lewis, ${ }^{6}$ Maik Haentschel'

'Division of Haematology, Oncology,

Rheumatology, Immunology and

Pulmonology, Department of Internal

Medicine, ${ }^{2}$ Department of Diagnostic and Interventional Radiology, ${ }^{3}$ Division of Endocrinology, Diabetology, Angiology, Nephrology and Clinical Chemistry, Department of Internal Medicine, Eberhard Karls University

Tuebingen, Tuebingen, Germany;

${ }^{4}$ Division of Pulmonology, University

Hospital Zurich, Zurich, Switzerland;

${ }^{5}$ Department of Pulmonary Diseases,

University Medical Center Groningen,

University of Groningen, Groningen,

the Netherlands; ${ }^{6}$ National Pollen and

Aerobiology Research Unit, Institute

of Science and the Environment,

University of Worcester, Worcester, UK

Correspondence: Maik Haentsche Division of Hematology, Oncology, Rheumatology, Immunology and

Pulmonology, Department of Internal Medicine, Eberhard Karls University

Tuebingen, Otfried-Müller-Straße I0,

D-72076 Tuebingen, Germany

$\mathrm{Tel}+49$ 707 I 29827 I।

Fax +49 707I 294598

Email maik.haentschel@med.

uni-tuebingen.de
Background: Lung volume reduction (LVR) improves breathing mechanics by reducing hyperinflation. Lobar selection usually focuses on choosing the most destroyed emphysematous lobes as seen on an inspiratory CT scan. However, it has never been shown to what extent these densitometric CT parameters predict the least deflation of an individual lobe during expiration. The addition of expiratory CT analysis allows measurement of the extent of lobar air trapping and could therefore provide additional functional information for choice of potential treatment targets.

Objectives: To determine lobar vital capacity/lobar total capacity (LVC/LTC) as a functional parameter for lobar air trapping using on an inspiratory and expiratory CT scan. To compare lobar selection by LVC/LTC with the established morphological CT density parameters.

Methods: 36 patients referred for endoscopic LVR were studied. LVC/LTC, defined as delta volume over maximum volume of a lobe, was calculated using inspiratory and expiratory CT scans. The CT morphological parameters of mean lung density (MLD), low attenuation volume (LAV), and 15 th percentile of Hounsfield units $(15 \% \mathrm{P})$ were determined on an inspiratory $\mathrm{CT}$ scan for each lobe. We compared and correlated LVC/LTC with MLD, LAV, and 15\%P.

Results: There was a weak correlation between the functional parameter LVC/LTC and all inspiratory densitometric parameters. Target lobe selection using lowest lobar deflation (lowest LVC/LTC) correlated with target lobe selection based on lowest MLD in 18 patients (50.0\%), with the highest LAV in 13 patients (36.1\%), and with the lowest $15 \% \mathrm{P}$ in 12 patients $(33.3 \%)$.

Conclusion: CT-based measurement of deflation (LVC/LTC) as a functional parameter correlates weakly with all densitometric $\mathrm{CT}$ parameters on a lobar level. Therefore, morphological criteria based on inspiratory CT densitometry partially reflect the deflation of particular lung lobes, and may be of limited value as a sole predictor for target lobe selection in LVR.

Keywords: emphysema, chronic obstructive pulmonary disease, high-resolution computed tomography, lung volume reduction

\section{Introduction}

Lung emphysema in patients with chronic obstructive pulmonary disease (COPD) is characterized by the abnormal and permanent expansion of distal airways and destruction of alveolar tissue. The resultant loss of elastic recoil causes an increase in air trapping and airway resistance.

Different techniques of endoscopic lung volume reduction (ELVR) in patients with severe lung emphysema have been extensively evaluated over the last 10 years 
to address these problems. Procedures such as valve and coil placement, glue, and steam application can improve lung function parameters, exercise tolerance, and quality of life. However, response to treatment is highly variable regardless of the technique used, as indicated by the high standard deviation of the evaluation parameters. ${ }^{1-6}$ This reflects clinical outcomes, where some patients have excellent treatment response, while others do not respond, or even deteriorate, after ELVR.

Although the degree of collateral ventilation and heterogeneity of emphysematous destruction have been identified as factors predicting success to valve treatment, ${ }^{1,2}$ treatment response remains highly variable. The current selection criteria for targeting lobes based solely on inspiratory highresolution CT (HRCT) and/or lung perfusion measurements are insufficient to predict treatment response.

The main aim of endoscopic and surgical therapy of lung emphysema is the reduction of hyperinflation. From a pathophysiological standpoint, the aim of treatment is to eliminate slow deflating lung areas and thus air trapping. Endoscopists would ideally wish to apply their various treatment methods to those lung areas which show least deflation during expiration. Previous studies have examined the degree of hyperfinflation on a for the whole lung and for both lungs separately; however, to our knowledge, this was never evaluated on a lobar level. ${ }^{7,8}$ Furthermore, it is unclear whether the degree of lung tissue destruction assessed on inspiratory CT predicts the limitation of deflation of a particular area.

This study evaluated whether the selection of a target area for lung volume reduction using densitometric criteria from the inspiratory HRCT correlates with a target area based on the degree of deflation during expiration.

\section{Material and methods}

\section{Patients}

36 patients with severe COPD referred for ELVR to the University Hospital in Tuebingen were examined by HRCT of the thorax in maximal inspiration and maximal expiration. We analyzed all consecutive patients with severe COPD between November 2011 and July 2014 who were referred to our department for lung volume reduction and who required a new CT scan of the thorax. Out of 43 patients, we have complete $\mathrm{CT}$ datasets in inspiration and expiration in 36 patients for further analysis.

\section{CT technique and image analysis}

All CT examinations were performed without intravenous contrast injection using the same 128-MDCT (Somatom
Definition AS+; Siemens Healthcare, Forchheim, Germany) with a $250-330 \mathrm{~mm}$ field of view, a $512 \times 512$ reconstruction matrix, and tube rotation time of $0.3 \mathrm{~s}$. CT scanning parameters were weight-adapted (120 kv, 100-120 mAs) and kept constant for both inspiratory and expiratory scans. All subjects were thoroughly coached in breathing techniques prior to the CT scan. They received standardized instructions for breath-holding at deep inspiration and end-tidal expiration during the spiral CT acquisition from apex to base of the lung. Axial images were reconstructed using the same slice thickness (range, 1-3 mm) and smooth reconstruction kernel (B31f or B35f) for inspiratory and expiratory CT scans.

The novel Syngo CT Pulmo 3D software (Siemens Healthcare) was used for the quantitative CT evaluation (CThistography) of the lung tissue. First, the three-dimensional CT data set was applied to automatic lung detection (threshold value, -500 Hounsfield unit [HU]) without major bronchi and vessels. Subsequently lung lobes were semi-automatically segmented using distance measurements between the lobar vessel trees and automatic fissure detection. All lobe borders were validated and, if necessary, corrected manually by an experienced physician. Finally, for each lobe (excluding the middle lobe, as it is not a target for lung volume reduction), mean lung density (MLD) in HU, low attenuation value (LAV) as percentage of lung tissue with HU below -950 , and 15 th percentile of $\mathrm{HU}(15 \% \mathrm{P})$ were calculated on maximal inspiration and maximal expiration.

\section{Functional and densitometic lobe selection criteria}

The functional parameter LVC/LTC (lobar vital capacity/ lobar total capacity - the amount of exhaled volume following full expiration in relation to the maximal inspiratory volume on a lobar level) was then calculated. This value is defined as delta volume over maximum volume and was calculated for each lobe separately as:

\section{$\underline{\text { Inspiratory volume - expiratory volume }}$ Inspiratory volume}

Functionally, hyperinflation of the entire lung is characterized by a high residual volume (RV)/total lung capacity (TLC) and a low vital capacity (VC)/TLC as determined by lung function testing, so in an analogous way we used CT scans in maximal inspiration and maximal expiration in order to measure vital capacity and total capacity on a lobar level (LVC and LTC, respectively). 
The densitometric criteria for selection of the target lobe were the lowest MLD, the highest LAV, and the lowest $15 \% \mathrm{P}$, respectively. These morphological criteria were applied both to inspiratory and to expiratory HRCTs. The lobe with the greatest degree of air trapping was considered to be the lobe with the lowest LVC/LTC, and this was defined as the target lobe.

The following lung function tests were measured using standardized lung function testing (Masterscreen PFT; Care Fusion, San Diego, CA, USA): forced expiratory volume in one second $\left(\mathrm{FEV}_{1}\right)$, forced vital capacity (FVC), RV, TLC in liters, ratio of RV to TLC calculated in percent, and carbon monoxide diffusion capacity in $\mathrm{mL} / \mathrm{min} / \mathrm{mmHg}$.

\section{Statistical methods}

We calculated the frequency that lobe selection based on measurement of LVC/LTC matched lobe selection based on densitometrically based inspiratory HRCT criteria. Spearman's correlation coefficients $\left(r_{s}\right)$ between LVC/LTC and inspiratory respectively expiratory HRCT parameters MLD, $\mathrm{LAV}$, and $15 \% \mathrm{P}$ were calculated. For statistical analysis, SAS jmp ${ }^{\circledR} 11.2$ (SAS, Cary, NC, USA) was used.

The study was approved by the ethics committee of the University Hospital of Tuebingen, Germany. All patients provided informed consent.

\section{Results}

HRCT data sets of 36 patients with severe COPD were studied. Demographic data of study population are shown in Table 1.

Lung function determined by spirometry and bodyplethysmography and CT-based LVC/LTC and lung densitometry are shown in Table 2 .

Table I Baseline characteristics of the patients

\begin{tabular}{ll}
\hline Characteristics & $\mathbf{N}=\mathbf{3 6}$ ( $100 \%)$ \\
\hline Age - years & \\
$\quad$ Mean \pm SD & $63.3 \pm 6.4$ \\
Gender $-\mathrm{n}(\%)$ & \\
$\quad$ Male & $21(58.3)$ \\
$\quad$ Female & $15(41.7)$ \\
Smoking state $-\mathrm{n}(\%)$ & \\
$\quad$ Nonsmoker & $1(2.8)$ \\
Current smoker & $9(25.0)$ \\
Ex-smoker & $24(66.7)$ \\
Unknown & $2(5.5)$ \\
Height $-\mathrm{cm}$ & \\
Mean \pm SD & $167.9 \pm 9.4$ \\
Weight $-\mathrm{kg}$ & \\
Mean \pm SD & $61.5 \pm 12.8$ \\
\hline
\end{tabular}

Note: Data presented as mean \pm SD or $\mathrm{n}(\%)$.
Table 2 Lung volumes and densitometry of the patients

\begin{tabular}{|c|c|c|}
\hline $\begin{array}{l}\text { Spirometric/bodyplethysmographic } \\
\text { determined lung function/volumes }\end{array}$ & & $N=36(100 \%)$ \\
\hline \multicolumn{3}{|l|}{$\mathrm{FEV}_{1}$ - liter } \\
\hline Absolute volume \pm SD & & $0.75 \pm 0.22$ \\
\hline Percentage of predicted value \pm SD & & $27.1 \pm 7.7$ \\
\hline \multicolumn{3}{|l|}{ FVC - liter } \\
\hline Absolute volume \pm SD & & $2.29 \pm 0.85$ \\
\hline Percentage of predicted value \pm SD & & $62.5 \pm 19.7$ \\
\hline \multicolumn{3}{|l|}{ RV - liter } \\
\hline Absolute volume \pm SD & & $5.88 \pm 1.46$ \\
\hline Percentage of predicted value \pm SD & & $264.7 \pm 71.7$ \\
\hline \multicolumn{3}{|l|}{ TLC - liter } \\
\hline Absolute volume \pm SD & & $8.21 \pm 1.68$ \\
\hline Percentage of predicted value \pm SD & & $134.9 \pm 24.5$ \\
\hline \multicolumn{3}{|l|}{$\mathrm{VC} / \mathrm{TLC}-\%$} \\
\hline Mean \pm SD & & $0.28 \pm 0.09$ \\
\hline \multicolumn{3}{|c|}{ Carbon monoxide diffusion capacity $-\mathrm{mL} / \mathrm{min} / \mathrm{mmHg}$} \\
\hline Absolute value \pm SD & & $2.79 \pm 0.86$ \\
\hline Percentage of predicted value \pm SD & & $32.66 \pm 8.86$ \\
\hline \multicolumn{3}{|l|}{$\begin{array}{l}\text { CT-based determined lung volumina } \\
\text { (based on all lobes of all patients) }\end{array}$} \\
\hline \multicolumn{3}{|l|}{ LVC/LTC - \% } \\
\hline Mean \pm SD & & $0.19 \pm 0.10$ \\
\hline $\begin{array}{l}\text { CT-based lung densitometry } \\
\text { (based on all lobes of all patients) }\end{array}$ & Inspiration & Expiration \\
\hline \multicolumn{3}{|l|}{ MLD - HU } \\
\hline Mean \pm SD & $-880 \pm 15.5$ & $-853 \pm 25.4$ \\
\hline \multicolumn{3}{|l|}{ LAV - percent $<-950 \mathrm{HU}$} \\
\hline Mean $\pm S D$ & $25.2 \pm 9.4$ & $17.9 \pm 9.3$ \\
\hline \multicolumn{3}{|l|}{$15 \% \mathrm{P}-\mathrm{HU}$} \\
\hline Mean $\pm S D$ & $-96 \mid \pm 12.4$ & $-95 I \pm 20.9$ \\
\hline
\end{tabular}

Note: Data presented as mean \pm SD.

Abbreviations: $15 \% \mathrm{P}$, 15th percentile of Hounsfield units; $\mathrm{FEV}_{1}$, forced expiratory volume in one second; FVC, forced vital capacity; HU, Hounsfield unit; LAV, low attenuation value; LTC, lobar total capacity; LVC, lobar vital capacity; MLD, mean lung density; RV, residual volume; TLC, total lung capacity; VC, vital capacity.

The first analysis focused on target lobe selection. Based on the volumetric measurements and the different densitometric parameters, the target lobes for either technique were determined. Then we compared how often lobe selection based on functional parameters matched with lobe selection based on densitometric ones. This was done on both inspiratory and expiratory HRCT scans. Lobe selection for lung volume reduction based on LVC/LTC matched with lobe selection based on MLD, LAV, and $15 \% \mathrm{P}$ in inspiratory HRCT in 18 (50.0\%), 13 (36.1\%), and 12 (33.3\%) cases, respectively. Lobe selection using LVC/LTC matched MLD, $\mathrm{LAV}$, and $15 \% \mathrm{P}$ in expiration in $18(50.0 \%), 16$ (44.4\%), and 17 (47.2\%) cases respectively. An example illustrating lobar volume, lobar densitometry, and their changes in inspiration and expiration are shown in Figure 1.

The second analysis evaluated the correlation between LVC/LTC and the different densitometric parameters in 

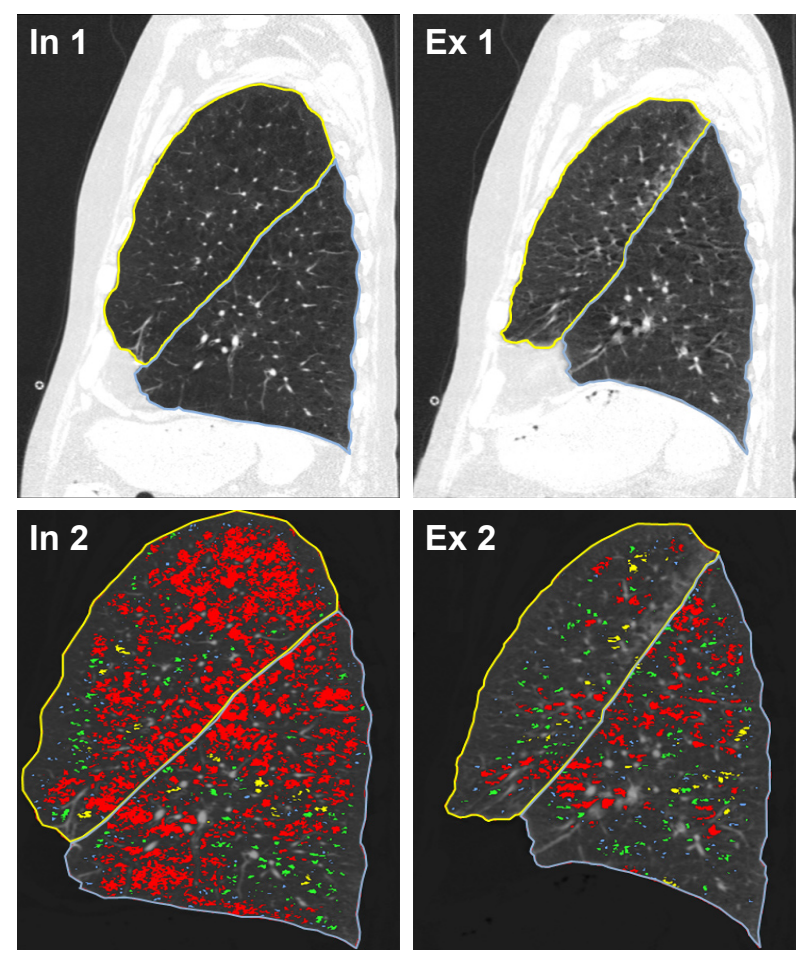

Figure I Example of a sagittal reconstruction of corresponding planes of the left lung in inspiration $(\ln I, \ln 2)$ and expiration (Ex I, Ex 2).

Notes: Although densitometric parameters in inspiration indicate a slightly higher degree of destruction in the UL in comparison to the LL (UL vs LL: MLD [HU] -889 vs $-88 \mathrm{I}$; LAV [\% <-950 HU] 18.4 vs 15.3 ; I5\% [HU] -949 vs -945$)$ there is a higher deflation during expiration in the UL compared to the LL (UL vs LL: LVC/LTC 0.43 vs 0.25 ). This example illustrates a huge diversity of volume changes between lobes with almost equal inspiratory densitometric parameters. Based on inspiratory densitometric parameters the target for lung volume reduction would have been the upper lobe. In contrast the lower deflation of the LL indicates to treat this lobe. Abbreviations: 15\% P, 15th percentile of Hounsfield units; HU, Hounsfield unit; LAV, low attenuation value; LL, lower lobe; LVC/LTC, lobar vital capacity/lobar total capacity; MLD, mean lung density; UL, upper lobe.

inspiration as well as in expiration. This analysis showed that correlation between LVC/LTC and inspiratory mean lung density, low attenuation value, and inspiratory $15 \% \mathrm{P}$ was weak. In contrast, there was a strong correlation between the three inspiratory densitometric parameters mean lung density, low attenuation value, and inspiratory $15 \% \mathrm{P}$ (Figure 2).

There was a moderate correlation between LVC/LTC and the expiratory densitometric parameters mean lung density, low attenuation value, and expiratory $15 \% \mathrm{P}$, whereas a strong correlation between the different expiratory densitometric parameters could be detected (Figure 3).

\section{Discussion}

COPD is an inflammatory disease which causes airway obstruction as a result of bronchitis, bronchiolitis, and lung emphysema (Figure 4). The destruction of lung parenchyma results in the loss of elastic recoil, which leads to an increased airway resistance due to the early collapse of the bronchi, tidal breathing at higher lung volumes, and finally ends in dyspnea through airflow limitation, hyperinflation, and a loss of gas exchanging surface. ${ }^{9}$ The extent of hyperinflation can be quantified by RV/TLC ratio or VC/TLC ratio. The increase of $\mathrm{RV}$ results in a decrease of $\mathrm{VC}$, which in turn results in a decrease in $\mathrm{FEV}_{1}$. In this situation, the reduction of $\mathrm{VC}$ seems to have a higher impact on $\mathrm{FEV}_{1}$ than the increase in airway resistance. ${ }^{9,10}$ Airway resistance, however, is further increased by bronchitis/bronchiolitis, and can reduce VC/TLC ratio as well. This effect can be so pronounced that patients with severe bronchiolitis can develop severe hyperinflation without any signs of emphysema.

The positive effect of lung volume reduction treatment in patients with severe emphysema is based mainly on reducing hyperinflation with resultant improvement of diaphragm and chest wall mechanics and a decrease in the work of breathing. An additional effect is the increase in elastic recoil, which increases expiratory airflow and lowers the lung volume at which tidal breathing takes place. Current studies in lung volume reduction have quantified the loss of function resulting from air trapping and hyperinflation by measurement of function of the whole lung, but lung function measurements at a lobar level have not been considered. However, lung volume reduction is usually focused on the treatment of a single lobe. In order to describe the differences between the lobes, current target lobe selection uses densitometry of inspiratory HRCT scans to assess the structural changes, namely the degree of emphysema and the amount of bronchiolitis.

Therefore, target lobe selection for lung volume reduction is routinely assessed by densitometric criteria based on inspiratory high-resolution chest CT scans. However, it has been shown that densitometric analyses of inspiratory CT imaging of the whole lung do not serve as a good predictor for functional limitations of emphysema. ${ }^{11}$ This is plausible since it can be assumed that hyperinflation, defined as elevated end-expiratory lung volume, cannot be determined accurately by densitometry in deep inspiration. This observation is in accordance with Omori et al, who found that inspiratory measurements assess the extent of emphysema and that expiratory measurements reflect airflow limitation and air trapping of the whole lung. ${ }^{12}$

Current target lobe selection in ELVR, based on densitometric data on inspiratory CT scans, assumes that the most emphysematous lobe has the least ventilation. The present study shows, however, that at a lobar level, densitometric data do not predict ventilation, ie, the assumption that the most emphysematous lobe shows the least ventilation is not valid. This difference between a morphological strategy on the one hand and a functional strategy on the other may explain 


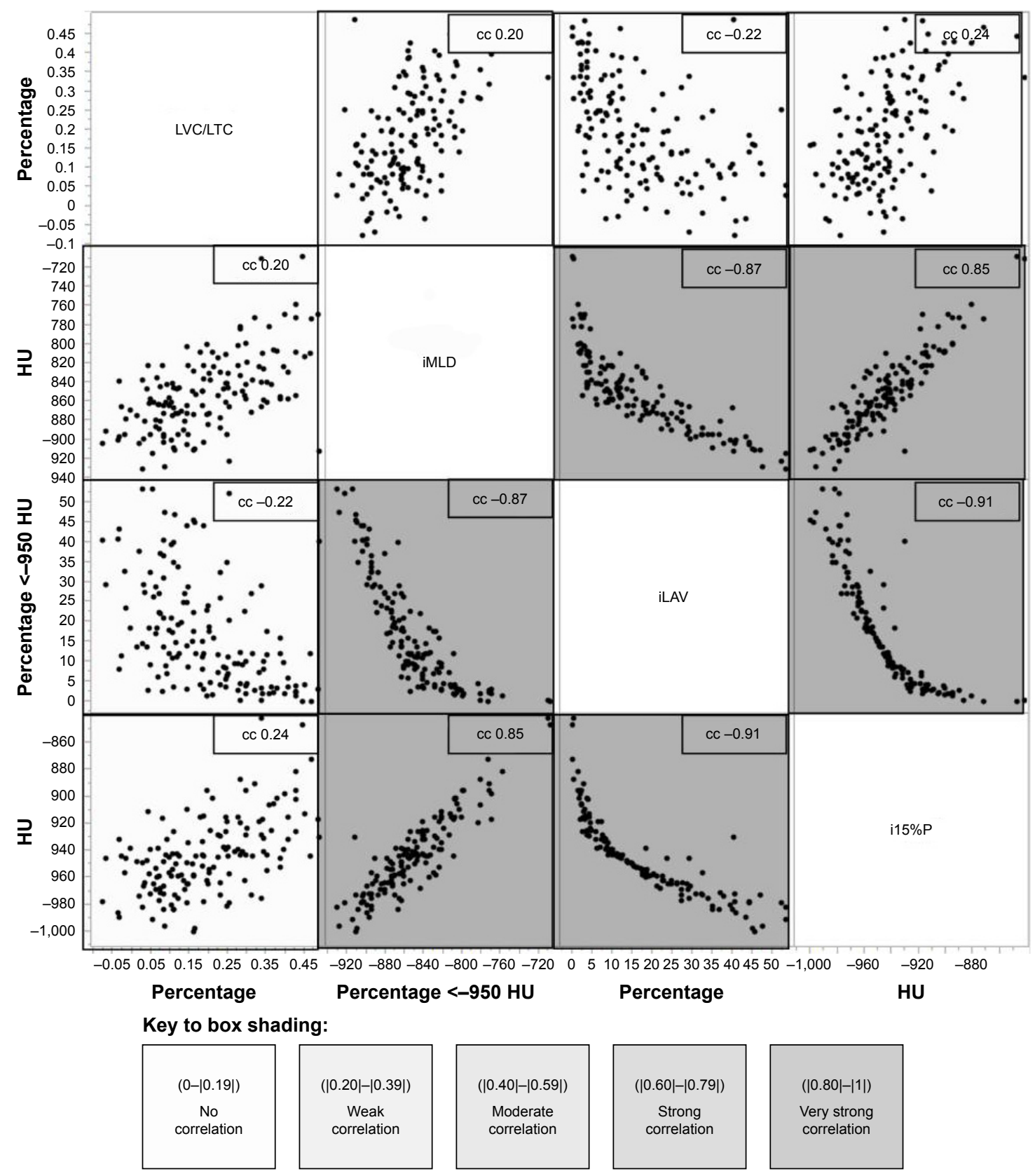

Figure 2 Correlation coefficients (cc) between LVC/LTC and iMLD, iLAV, and il5\%P [HU] in different shading, indicating the grade of correlation. Notes: Every dot represents one lobe. Parameters of all four measured lobes (right UL, right LL, left UL, and left LL) of every patient are given.

Abbreviations: $\mathrm{HU}$, Hounsfield unit; i I5\%P, inspiratory 15 th percentile of Hounsfield units; iLAV, inspiratory low attenuation value; iMLD, inspiratory mean lung density; LL, lower lobe; LVC/LTC, lobar vital capacity/lobar total capacity; UL, upper lobe.

why variation in treatment response between patients with comparable basic and morphological conditions is so often observed. In contrast to inspiratory chest CT measurements, lobar densitometric parameters in expiratory CT scans correlate better with LVC/LTC. This indicates that densitometric and functional analyses at a lobar level mirror the discrepancies noted with analyses of the whole lung.

However, it has to be considered that in our study only patients with COPD and severe lung emphysema were studied. In patients with severe hyperinflation due to pure bronchiolitis without emphysema, the use of functional parameters alone might theoretically indicate that lung volume reduction treatment may be of benefit, although, clearly, this is not the case. Thus, in our opinion functional measurements should be combined with densitometric CT assessment of the lung parenchyma. This allows for differentiation between patients with low deflation but low tissue destruction, due to small airways disease, and patients with emphysema who have high tissue destruction but the same low deflation volumes. 


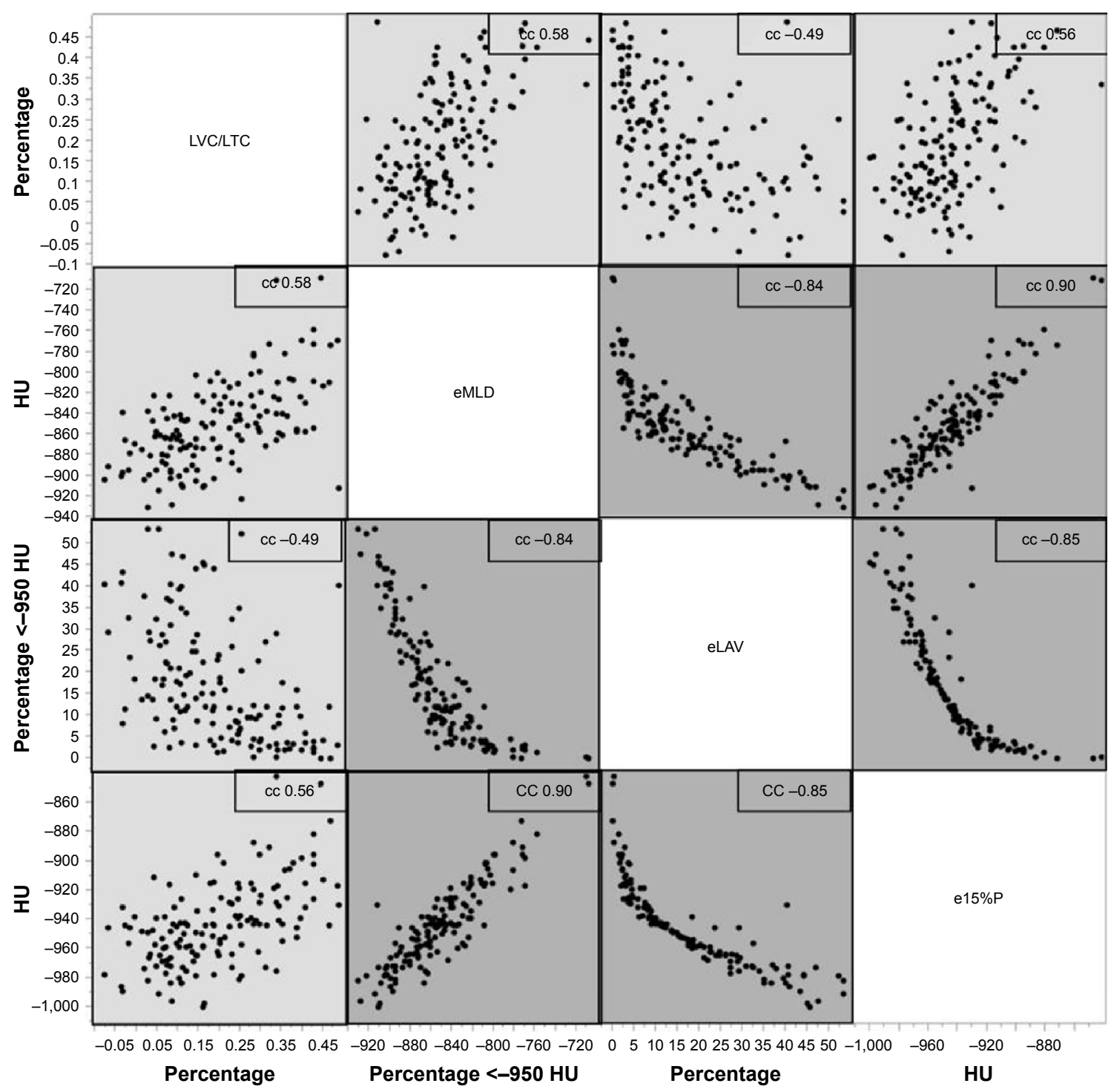

Key to box shading:
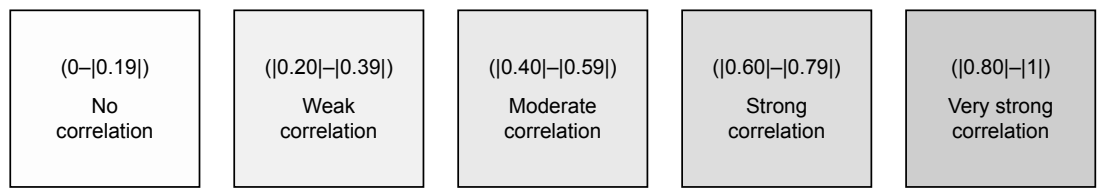

Figure $3 \mathrm{cc}$ between LVC/LTC and eMLD, eLAV, and el5\%P [HU] in different shading, indicating the grade of correlation.

Notes: Every dot represents one lobe. Parameters of all four measured lobes (right UL, right LL, left UL, and left LL) of every patient are given.

Abbreviations: cc, correlation coefficient; eI5\%P, expiratory I5th percentile of Hounsfield units; eLAV, expiratory low attenuation value; eMLD, expiratory mean lung density; HU, Hounsfield unit; LL, lower lobe; LVC/LTC, lobar vital capacity/lobar total capacity; UL, upper lobe.

Therefore, one can speculate that functional parameters in patients with morphological evidence of emphysema will improve target selection. Although the CT-based parameter, lobar LVC/LTC, reflects functional aspects on a lobar level in our study and goes along with the current concept of lung volume reduction, its ability to predict treatment response has not yet been evaluated. This needs to be confirmed in a prospective trial with clinical endpoints which compare a morphological based approach with a morphological plus functional approach for target lobe selection. Other limitations of the study are the small number of evaluated patients, which additionally weakens a further explorative analysis and the inconsistent timing between bronchodilator application and CT scanning.

A dedicated software easily allows to calculate lobar volumetric and lobar densitometric parameters from CT 


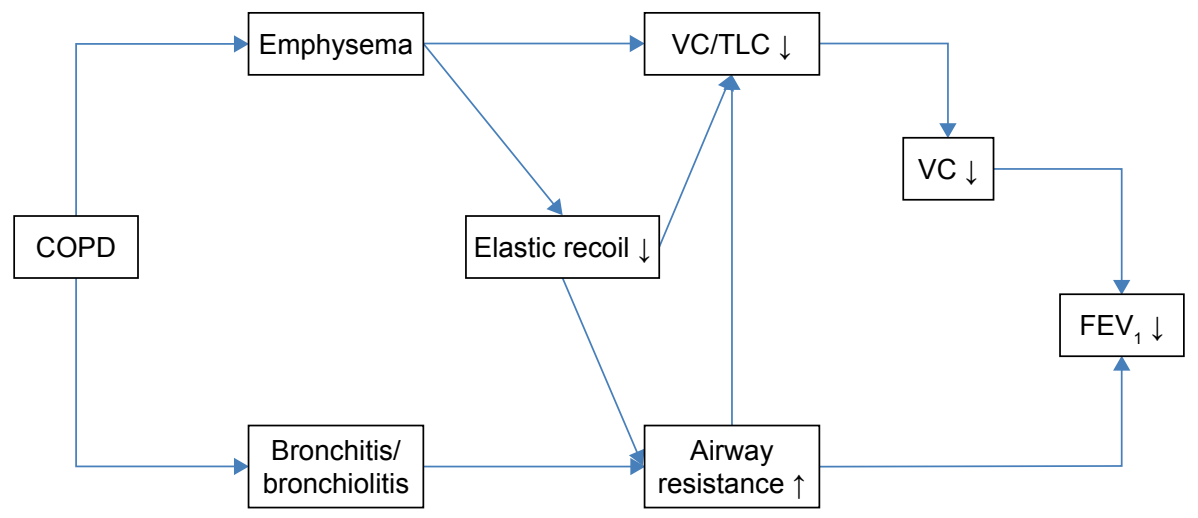

Figure 4 Pathophysiology of emphysema.

Note: $\downarrow$ means decrease and $\uparrow$ means increase.

Abbreviations: COPD, chronic obstructive pulmonary disease; FEV , forced expiratory volume in the first second; TLC, total lung capacity; VC, vital capacity.

scans of the thorax. Instead of using the absolute volume change in each lobe as a criterion, we have chosen the relative parameter LVC/LTC for two reasons. First, the chest-CTbased densitometric parameters are relative parameters too, and the comparison of an absolute parameter with a relative parameter would result in a methodological problem. Second, due to the different size of the lobes, normal values for the expiratory volume would be dissimilar.

As in clinical practice, the middle lobe is not chosen as a single target for lung volume reduction, therefore we excluded the middle lobe from the evaluation.

The present study analyzed function and tissue density at a lobar level. But not all lung volume reduction techniques, such as classical lung volume reduction surgery, treat whole lobes. Therefore, it might be questionable whether these lobar-based values should serve as selection criteria for therapies which do not treat a whole lobe. However, this study shows the discordance of densitometrical changes and function parameters, and this may be not only relevant in comparing different lobes in their entirety; this difference may be also relevant within a single lobe.

In clinical reality, the choice of a target lobe for interventional lung volume reduction is complex, and many factors may play a role. The current study illustrates that HRCT-based inspiratory densitometric parameters only partially reflect the function of certain lung areas. The distribution of hyperinflation, which reflects the local loss of ability to expire, seems to play an important role too. Lung volume reduction is based on pathophysiological principles, and the current basis of target lobe selection by morphological criteria on inspiratory CT scans is an insufficient predictor of treatment response, and additional functional methods need to be considered.

\section{Disclosure}

The authors report no conflicts of interest in this work.

\section{References}

1. Sciurba FC, Ernst A, Herth FJ, et al. A randomized study of endobronchial valves for advanced emphysema. N Engl J Med. 2010;363: 1233-1244.

2. Herth FJ, Noppen M, Valipour A, et al. Efficacy predictors of lung volume reduction with Zephyr valves in a European cohort. Eur Respir J. 2012;39:1334-1342.

3. Davey C, Zoumot Z, Jordan S, et al. Bronchoscopic lung volume reduction with endobronchial valves for patients with heterogeneous emphysema and intact interlobar fissures (the BeLieVeR-HIFi study): a randomized controlled trial. Lancet. 2015;386:1066-1073.

4. Slebos DJ, Hartman JE, Klooster K, et al. Bronchoscopic coil treatment for patients with severe emphysema: A meta-analysis. Respiration. 2015;90:136-145.

5. Hartman JE, Klooster K, Ten Hacken NH, Slebos DJ. Treatment of emphysema using bronchoscopic lung volume reduction coil technology: an update on efficacy and safety. Ther Adv Respir Dis. 2015;9: 251-259.

6. Choi M, Lee WS, Lee M, et al. Effectiveness of bronchoscopic lung volume reduction using unilateral endobronchial valve: a systematic review and meta-analysis. Int J Chron Obstruct Pulmon Dis. 2015;10: 703-710.

7. Kim EY, Seo JB, Lee HJ, et al. Detailed analysis of the density change on chest CT of COPD using non-rigid registration of inspiration/ expiration CT scans. Eur Radiol. 2015;25:541-549.

8. Eberhardt R, Gompelmann D, Herth FJ, Schuhmann M. Endoscopic bronchial valve treatment: patient selection and special considerations. Int J Chron Obstruct Pulmon Dis. 2015;10:2147-2157.

9. Fessler HE, Scharf SM, Ingenito EP, McKenna RJ Jr, Sharafkhaneh A. Physiologic basis for improved pulmonary function after lung volume reduction. Proc Am Thorac Soc. 2008;5:416-420.

10. Fessler HE, Permutt S. Lung volume reduction surgery and airflow limitation. Am J Respir Crit Care Med. 1998;157:715-722.

11. Washko GR, Criner GJ, Mohsenifar Z, et al. Computed tomographicbased quantification of emphysema and correlation to pulmonary function and mechanics. COPD. 2008;5:177-186.

12. Omori H, Fujimoto K, Katoh T. Computed-tomography findings of emphysema: correlation with spirometric values. Curr Opin Pulm Med. 2008;14:110-114. 


\section{Publish your work in this journal}

The International Journal of COPD is an international, peer-reviewed journal of therapeutics and pharmacology focusing on concise rapid reporting of clinical studies and reviews in COPD. Special focus is given to the pathophysiological processes underlying the disease, intervention programs, patient focused education, and self management protocols.

This journal is indexed on PubMed Central, MedLine and CAS. The manuscript management system is completely online and includes a very quick and fair peer-review system, which is all easy to use. Visit http://www.dovepress.com/testimonials.php to read real quotes from published authors.

Submit your manuscript here: http://www.dovepress.com/international-journal-of-chronic-obstructive-pulmonary-disease-journal 